\title{
An $n$-in-a-row type game
}

\author{
Joshua Erde \\ Fachbereich Mathematik \\ Bundesstr. 55 \\ 20146, Hamburg, Germany \\ joshua.erde@uni-hamburg.de
}

\author{
Mark Walters \\ School of Mathematical Sciences \\ Queen Mary University of London \\ London E1 4NS, United Kingdom \\ m.walters@qmul.ac.uk
}

Submitted: Jan 7, 2015; Accepted: Jul 4, 2016; Published: Jul 22, 2016
Mathematics Subject Classifications: 91A46

\begin{abstract}
We consider a Maker-Breaker type game on the square grid, in which each player takes $t$ points on their $t^{\text {th }}$ turn. Maker wins if he obtains $n$ points on a line (in any direction) without any of Breaker's points between them. We show that, despite Maker's apparent advantage, Breaker can prevent Maker from winning until about his $n^{\text {th }}$ turn. We actually prove a stronger result: Breaker only needs to claim $\omega(\log t)$ points on his $t^{\text {th }}$ turn to prevent Maker from winning until this time. We also consider the situation when the number of points claimed by Maker grows at other speeds, in particular, when Maker claims $t^{\alpha}$ points on his $t^{\text {th }}$ turn.
\end{abstract}

Keywords: Maker-Breaker; $n$-in-a-row game

\section{Introduction}

The unrestricted $n$-in-a-row game is a Maker-Breaker game played on $\mathbb{Z}^{2}$, where two players, Maker and Breaker, take turns claiming unclaimed points in $\mathbb{Z}^{2}$. Maker wins if he can claim $n$ consecutive points in a row either vertically, horizontally, or diagonally. It is known that when $n \leqslant 4$ Maker has a winning strategy, whereas for $n \geqslant 8$ Breaker has a strategy that prevents Maker from ever winning [6]. It will be convenient to consider the game as consisting of a series of rounds, each of which consists of one of Maker's turns and the subsequent turn of Breaker.

Erde [3] considered a variation of this game where, instead of claiming one point each turn, the number of points claimed by a player on his $t^{\text {th }}$ turn is a function of $t$. In particular, suppose on their first turn Maker and Breaker each claim 1 point, but on their second turn they each claim 2 points, and 3 on their third, and so on. Unlike the $n$-in-a-row game this game is clearly never a Breaker win, since on his $n^{\text {th }}$ turn Maker can claim an entire winning line. However, Erde showed that Maker cannot win this game in time less than $(1-o(1)) n$ (i.e., before the $(1-o(1)) n^{\text {th }}$ round). 
Beck [2] considered a generalisation of the unrestricted $n$-in-a-row game in which the winning lines are allowed to have arbitrary slopes; that is, a Maker-Breaker game on $\mathbb{Z}^{2}$ where Maker wins if he can claim $n$ consecutive points in a line, with any slope. Clearly this game is easier for Maker than the $n$-in-a-row game. Indeed Beck showed that this game is a Maker win for all $n$ (recall that the ordinary game is known to be a Breaker win for all $n \geqslant 8$ ). Since this game is easier for Maker it raises the possibility that the analogous modified version where the number of points claimed on each turn is increasing can be won by Maker in time less than $(1-o(1)) n$.

In fact, we consider an even easier game for Maker. The players take turns claiming points in $\mathbb{Z}^{2}$, with each player claiming $t$ points on their $t^{\text {th }}$ turn. Maker wins if he gets $n$ points on a straight line (in any direction) with no point of Breaker's between the first and last of these $n$. The points need not be consecutive and there may be other points of Maker or Breaker on this line. (Note this is not a standard Maker-Breaker game as Maker's winning sets depend on Breaker's points.) We call such a line segment a winning line segment. If each player claimed instead a fixed number of points in their turn, this game would be a slight variant of the Kaplansky $n$-in-a-line game considered previously by Kleitman and Rothschild [4] and Beck [1]. As before, Maker can claim the whole of a winning line segment on his $n^{\text {th }}$ turn and so he can definitely win by time $n$. Our first result is that, even in this game, this is essentially the best Maker can do.

Theorem 1. In the above game there is a strategy for Breaker which prevents Maker from winning before time $(1-o(1)) n$.

Remark. Throughout the paper we use the standard notations $O, o$ and $\Omega$ as well as the common, but less standard, notation $f=\omega(g)$ which denotes the property that $\lim _{n \rightarrow \infty} f(n) / g(n)=\infty$.

We also extend Theorem 1 substantially. For functions $m, b: \mathbb{N} \rightarrow \mathbb{N}$ define the $(m, b)$ $n$-in-a-row game as follows. Maker claims $m(t)$ points on his $t^{\text {th }}$ turn and Breaker claims $b(t)$ points on his. For simplicity we shall assume that both $m(t)$ and $b(t)$ are monotone increasing. As before, Maker wins when he has claimed a winning line segment. Obviously, if $m(t) \geqslant n$ then Maker can win on his $t^{\text {th }}$ turn. In this paper we are primarily interested in the question of for which $m$ and $b$ Maker can win significantly sooner than this.

Definition. The winning time of the $(m, b)$-n-in-a-row game is the earliest time by which Maker can guarantee to win; i.e., Maker can win by this time regardless of Breaker's play. We will denote this quantity by $\tau_{n}(m, b)$, or just $\tau_{n}$ when $m$ and $b$ are obvious from context.

Our main result is that, under a wide range of conditions, Maker cannot win much before the time when $m(t) \geqslant n$.

Theorem 2. Let $m(t)=t^{\alpha}, b$ be a function, and, for all $n \geqslant 1$, let $\tau_{n}$ be the winning time for the $(m, b)$-n-in-a-row game.

1. If $\alpha \geqslant 1$ and $b(t)=\omega(\log t)$ then $m\left(\tau_{n}\right)=(1+o(1)) n$.

2. If $\alpha<1$ and $b(t)=\omega\left(t^{1-\alpha}\right)$ then $m\left(\tau_{n}\right)=(1+o(1)) n$. 
In contrast we have the following theorem, which show that the requirement on $b$ in Theorem 2 is best possible in the case $\alpha \geqslant 1$.

Theorem 3. Let $m(t)=t^{\alpha}$ with $\alpha>0$ and $b=O(\log t)$. Then there exists some $\varepsilon>0$ such that $m\left(\tau_{n}\right) \leqslant(1-\varepsilon) n$. Furthermore, if $b=o(\log t)$ then $m\left(\tau_{n}\right)=o(n)$.

Remark. We remark that our strategy for Maker actually gives a horizontal line of $n$ consecutive points, and so it also a winning strategy in the variant of the unrestricted $n$-in-a-row game with these parameters.

For $\alpha<1$ the bounds on $b$ in Theorems 2 and 3 are very different. However, our strategy for Breaker in Theorem 2 has a special form: Breaker never relies on claiming a single point on two of Maker's lines. More precisely, Breaker can still delay Maker's win in all the cases of Theorem 2 if, whenever he claims any point, he also has to designate a specific direction, and the point only breaks Maker's winning sets through that point in that specific direction. (Breaker may claim the same point with a different direction but that counts as an extra point.) We call the version of the game where Breaker has to specify this direction the $(m, b)$-directed-Breaker game. We also show that, for $\alpha<1$, the bound for $b$ given by Theorem 2 is essentially tight for this game.

Theorem 4. Suppose that $m(t)=t^{\alpha}$ for $\alpha<1$ and $b(t)=o\left(t^{1-\alpha}\right)$. Let $\tau_{n}$ be the winning time for $(m, b)$-directed-Breaker $n$-in-a-row game. Then $m\left(\tau_{n}\right)=o(n)$.

\section{Elementary remarks}

We start with a trivial remark. We can think of the points that Breaker has claimed at any time in the game as having split each line in the plane into a number of line segments: blocks of points that are unclaimed or claimed by Maker, either lying in between two of Breaker's points, or one of Breaker's points and infinity (i.e., an infinite ray), or a line not containing any of Breaker's points. We call any such segments that do not contain at least $n$ integer points, claimed or otherwise, inactive. Inactive line segments are not useful to Maker as they cannot be extended to a winning line segment. We call all other line segments (i.e., line segments that are not inactive) active.

Suppose that Maker wins the $(m, b)$-n-in-a-row game on his $T^{\text {th }}$ turn. Then, after Breaker's last turn there must have been an active line segment containing at least $n-$ $m(T)$ of Maker's points. In particular, if $m(T)<(1-\varepsilon) n$ for some $\varepsilon>0$, then there must have been an active line segment containing at least $\varepsilon n$ of Maker's points.

We use this observation to relate our game to a simpler game that we will mention at times. In the game as described so far there are advantages and disadvantages to playing first: if a player claims a point then it stops his opponent from claiming it (an advantage) but means his opponent knows where he has played (a disadvantage).

In the modified version, which we will call the batched $(m, b)$ - $n$-in-a-row game, first Maker chooses some number of rounds $T$ and claims all the points he would have claimed in the $(m, b)$-n-in-a-row game in those $T$ rounds. Then Breaker claims all of his points from those $T$ rounds, with the additional freedom that he may choose points that Maker 
has already chosen. Maker wins if his largest active line segment at the end of the game has size at least $n-m(T)$. The winning time of the game is the smallest $T$ such that Maker can guarantee to win. We will usually denote it by $\sigma_{n}$.

Observe that if Maker wins the batched game, the position at the end of that game is one where, in the normal game, Maker could win on his next move. Since Maker can do at least as well in the normal game, an immediate consequence of this observation is that $\tau_{n} \leqslant \sigma_{n}+1$. Thus a strategy of Maker's for the batched game immediately gives a strategy of Maker's for the normal game. This can be useful, as the batched game is much easier to think about.

The key tool in our proofs will be the Szemerédi-Trotter Theorem [5], and a simple corollary of it.

Theorem (Szemerédi-Trotter). Suppose $P$ is a set of points, $L$ is a set of line segments in $\mathbb{R}^{2}$ such that any two line segments meet in at most one point, and let $I$ be the set of incidences (an incidence is a point-line segment pair with the line segment containing that point). Then

$$
|I|=O\left(|P|^{2 / 3}|L|^{2 / 3}+|P|+|L|\right) .
$$

Corollary (Szemerédi-Trotter). Let $P, L$ be as above. Then the number of line segments containing at least $k$ points is

$$
O\left(\frac{|P|^{2}}{k^{3}}+\frac{|P|}{k}\right)
$$

We remark that this theorem is normally stated in terms of lines rather than line segments, however it is a folklore result that the line segment version is implied as a simple consequence.

As we will be using the Szemerédi-Trotter Theorem it is convenient to make the following definition.

Definition. For any $n$ and $k$ define $S z T(n, k)$ to be the maximum number of incidences that can occur between $n$ points and $k$ (non-overlapping) line segments.

Next we illustrate our methods by applying them to the batched game.

Proposition 5. Let $m(t)=t, b(t)=\omega(1)$ and $\sigma_{n}$ be the winning time for the batched $(m, b)$-n-in-a-row game. Then $\sigma_{n}=(1+o(1)) n$.

Proof. Let $\varepsilon>0$. We will show that, for all $n$ sufficiently large, if Maker chooses to play for $T \leqslant(1-\varepsilon) n$ rounds, then Breaker has a winning strategy. This will show that $\sigma_{n} \geqslant(1-\varepsilon) n$ for all sufficiently large $n$, which implies the result.

In the normal game, Maker would claim at most $T^{2}$ points up to time $T$ so, in the batched game, he chooses at most $T^{2}$ points. We may assume that $T \geqslant \sqrt{n}$, as otherwise Maker has claimed fewer than $n$ points and so cannot win.

Let us consider the set of lines in $\mathbb{Z}^{2}$ containing any of Maker's points. If any of these lines contains more than $n$ of Maker's points, we artificially split it into a number 
of disjoint line segments each containing at most $n$ points. In this way we have a set $P$ of at most $T^{2}$ points and a set $L$ of line segments in $\mathbb{Z}^{2}$ satisfying the conditions of the corollary to the Szemerédi-Trotter Theorem.

This theorem tells us that the number of line segments containing more than $\varepsilon n$ points is (since $T \leqslant n$ ) at most

$$
O\left(\frac{T^{4}}{(\varepsilon n)^{3}}+\frac{T^{2}}{\varepsilon n}\right)=O(T) .
$$

Since Breaker is allowed to claim points that Maker has already claimed, he can split each of these line segments into subsegments containing at most $\varepsilon n / 2$ of Maker's points by claiming one in every $\varepsilon n / 2$ points of Maker's. Since each line segment contained at most $n$ of Maker's points, Breaker only has to claim at most $4 / \varepsilon$ points in each line segment.

Therefore, since $b(t)=\omega(1)$, Breaker has $\omega(T)$ points to claim, and so has sufficiently many points to follow this strategy in each of the $O(T)$ line segments. Clearly this is a winning strategy for Breaker as claimed.

Returning to the normal game, suppose that $m\left(\tau_{n}\right) \leqslant(1-\varepsilon) n$ for some $\varepsilon>0$ (where as usual $\tau_{n}$ is the winning time of the $(m, b)$ - $n$-in-a-row game). Then, as observed above, after Breaker's last turn, there must have been an active line segment containing at least $\varepsilon n$ of Maker's points. Thus, we see that it is important for Breaker to try to ensure that no such line segments exist at the end of his turn.

Definition. Suppose that $\ell$ is a line segment containing some of Maker's points. We say that Breaker has $\varepsilon$-split the line segment $\ell$ if he has claimed points in it splitting it into smaller line segments such that, after the split, there are no active segments containing (at least) $\varepsilon n$ of Maker's points. In cases where $\varepsilon$ is clear we will just say that Breaker has split the line segment.

The following lemma provides a simple bound on how many points Breaker needs to split a line.

Lemma 6. Suppose that $\varepsilon>0$ and that $\ell$ is an active line segment containing fewer than $n$ of Maker's points. Then Breaker can $\varepsilon$-split $\ell$ using at most $2 / \varepsilon$ points.

Proof. Breaker starts from one end and counts along $\varepsilon n-1$ of Maker's points. He would like to claim the next integer point, say $x$, on the line but this may not be possible as $x$ may already be a Maker point. So instead he claims both the last free integer point before $x$ on the line segment and the first free integer point after $x$. He then repeats the process on the remaining line segment after the second of these points. Obviously when this process has finished there is no active line segment containing $\varepsilon n$ of Maker's points and Breaker has claimed at most $2 / \varepsilon$ points. 


\section{A weighted bin game}

In this section we introduce a weighted bin game. This is a simpler game that is easy to analyse but our main proofs will compare the real game with this simple game. It is a single player (who we will call Maker) game.

Definition. Suppose $T$ is a constant and $b, M: \mathbb{N} \rightarrow \mathbb{N}$ are functions. The weighted bin game $(b, M, T)$ is the following game. The game is played with $1+\sum_{t=1}^{T} b(t)$ bins. On turn $t$ Maker adds some weight (a non-negative real number) to some bins subject to some constraints given below. Then the $b(t)$ largest bins are removed. The game lasts $T$ turns after which there is a single remaining bin. Maker's aim is to maximise the weight of this bin.

The constraint for Maker is the following: for any $s>0$ the total weight added during the last $s$ turns is at most $M(s)$.

Lemma 7. Suppose that Maker plays the game above playing weight $w_{i}$ on his $i^{\text {th }}$ turn for each $i$. Then the weight remaining in the last bin is at most

$$
\sum_{s=1}^{T} \frac{w_{s}}{\sum_{t=s}^{T} b(t)+1} .
$$

Proof. Suppose at the start of his $s^{\text {th }}$ turn the $\sum_{t=s}^{T} b(t)+1$ remaining bins have average weight $a$. Clearly, after Maker adds this turn's weight, the average weight is

$$
a+\frac{w_{s}}{\sum_{t=s}^{T} b(t)+1} .
$$

and this average weight does not increase when the $b(t)$ largest bins are removed.

Hence, at the end of the game there is a single bin with (average) weight at most

$$
\sum_{s=1}^{T} \frac{w_{s}}{\sum_{t=s}^{T} b(t)+1}
$$

as claimed.

Remark. Obviously Maker can obtain the bound given in this lemma, but we shall not make use of that.

Lemma 8. Suppose that $T$ is fixed, that $M(s)$ is any increasing function $\mathbb{N} \rightarrow \mathbb{N}$ with $M(0)=0$, and that $b(t)$ has the property that, for any $s$, we have $\sum_{t=s}^{T} b(t) \geqslant b(T)(T-$ $s+1) / 2$. Let $\Delta M$ be the function given by $\Delta M(s)=M(s)-M(s-1)$. Then any strategy for the weighted bin game $(b, M, T)$ finishes with weight at most

$$
\frac{2}{b(T)} \sum_{t=1}^{T} \frac{\Delta M(t)}{t}
$$

in the final bin. 
Remark. The constraint on $b$ is roughly requiring that $b$ is not super-linear in growth.

Proof. Suppose Maker plays weight $w_{t}$ on the $t^{\text {th }}$ turn. By the previous lemma the weight in the final bin is at most

$$
\sum_{s=1}^{T} \frac{w_{s}}{\sum_{t=s}^{T} b(t)+1}
$$

The constraint on Maker is that, for any $s$, in the last $s$ turns he adds at most $M(s)$ weight to the bins, and so, for each $s$

$$
\sum_{t=s}^{T} w_{t} \leqslant M(T-s+1) .
$$

Since the denominators in $(*)$ are fixed and decreasing, it is clear that to maximise this quantity we want to add the weight in as late a turn as possible. Thus the largest (*) can be is if we chose to add weight $w_{s}=\Delta M(T-s+1)$ for each $s$.

To conclude the proof we just have to bound $(*)$ in this case. We have that

$$
\sum_{s=1}^{T} \frac{w_{s}}{\sum_{t=s}^{T} b(t)+1} \leqslant \sum_{s=1}^{T} \frac{\Delta M(T-s+1)}{\frac{1}{2} b(T)(T-s+1)+1} \leqslant \frac{2}{b(T)} \sum_{t=1}^{T} \frac{\Delta M(t)}{t}
$$

as claimed.

\section{Proof of Theorem 2}

Proof. Let $\varepsilon>0$. The proof breaks down into three pieces: first we give the strategy that Breaker will follow. Then, we show that Breaker is able to follow this strategy. Finally we show that, if Breaker follows this strategy, then, for all sufficiently large $n$, Maker cannot win before time $t$ such that $m(t)=(1-\varepsilon) n$. It will follow that $m\left(\tau_{n}\right) \geqslant(1-\varepsilon) n$ for all sufficiently large $n$ which implies the result.

Observe that replacing $b$ by a smaller function only makes things harder for Breaker. Thus we may assume that, for all sufficiently large $t$ and any $1 \leqslant s \leqslant t$, we have $\sum_{i=s}^{t} b(i) \geqslant b(t)(t-s+1) / 2$ (whilst maintaining $b$ being $\omega(\log t)$ or $\omega\left(t^{1-\alpha}\right)$ as appropriate). This will be useful when we apply Lemma 8 later.

Let $\hat{b}(t)=\frac{\varepsilon}{4} b(t)$. Breaker's strategy is as follows: on his $t^{\text {th }}$ turn Breaker $\varepsilon / 2$-splits the $\hat{b}(t)$ active line segments containing the most points of Maker.

Breaker can follow this strategy since, assuming Maker has not already won, there were no active line segments with more than $n$ points at the end of Makers turn. Thus, by Lemma 6 these $\hat{b}$ line segments can be split using at most $b$ points.

Now, suppose for the sake of contradiction that Maker can win against this strategy in time $T+1$ such that $m(T+1) \leqslant(1-\varepsilon) n$. We will obtain the desired contradiction as follows: First we will use Maker's play against this strategy to give a winning play for the weighted bin game (with certain parameters). Then, we will use Lemma 8 and the 
Szemérdi-Trotter Theorem to deduce a bound relating $b, T$ and $n$. Finally, some simple calculations will yield the contradiction.

Before proceeding further we note that $T$ must tend to infinity with $n$ as Maker cannot win the $n$-in-a-row game before he has claimed at least $n$ points in total.

\section{Step 1}

We will show that there is a winning strategy for Maker in the weighted bin game $(\hat{b}, M, T)$, where $M(s)=\operatorname{SzT}\left(T^{\alpha} s, \hat{b}(T) s+1\right)$, giving at least weight $\varepsilon n / 2$ in the remaining bin.

First observe that, since Maker wins on turn $T+1$ and $m(T+1) \leqslant(1-\varepsilon) n$, after Breaker's $T^{\text {th }}$ turn Maker must have had an active line segment containing at least $\varepsilon n$ points.

Define $B(s)=\sum_{t=T-s+1}^{T} \hat{b}(t)$. Consider the $B(T)+1$ line segments given by the $B(T)$ segments that Breaker splits during the game together with Maker's winning line segment.

We create a bin $Q_{i}$ corresponding to each line segment $L_{i}$. We map a situation in the $n$-in-a-row game to a situation in the weighted bin game as follows: place weight $\ell_{i}-\varepsilon n / 2$ in bin $Q_{i}$ where $\ell_{i}$ is the number of points on line segment $L_{i}$, placing weight zero if this is negative.

Note we do not consider the line segment as being 'created' until Breaker has claimed its endpoints: in particular if Maker claims some points on a line segment it only adds weight to the bin corresponding to the current segment, not sub-segments that will be created later. This does not matter because, by the definition of splitting a line, all the newly created line segments have at most $\varepsilon n / 2$ points, and so map to empty bins. Thus Breaker's move in the $n$-in-a-row game under the strategy defined above corresponds to removing the $\hat{b}$ heaviest bins in the weighted bin game.

After the $T^{\text {th }}$ round in the $n$-in-a-row game the remaining line segment has at least $\varepsilon n$ points so the corresponding bin has at least weight $\varepsilon n / 2$.

The final part of this step is to check the bound on $M$ : that is we show that Maker does not add more than $M(s)=\operatorname{SzT}\left(T^{\alpha} s, \hat{b}(T) s+1\right)$ in his last $s$ rounds of the weighted bin game. Consider the last $s$ rounds in the $n$-in-a-row game. In these turns the weight added in the weighted bin game is (at most) the number of new incidences in the $n$-in-arow game. The total number of points added in these turns is at most $T^{\alpha} s$ and there are $B(s)+1 \leqslant \hat{b}(T) s+1$ remaining line segments. Since we are only counting the current line segments these segments only intersect in single points, so the Szemerédi-Trotter Theorem does apply.

The number of points added to these $\hat{b}(T) s+1$ line segments is at most the number of points on the largest $\hat{b}(T) s+1$ line segments through these (at most) $T^{\alpha} s$ points. By definition this is at most $\operatorname{SzT}\left(T^{\alpha} s, \hat{b}(T) s+1\right)$ which concludes this step.

\section{Step 2}

In the previous step we showed that Maker has a strategy for the $(\hat{b}, M, T)$ weighted bin game, giving at least weight $\varepsilon n / 2$ in the last remaining bin. In this step we combine this with our bounds for when Maker can win the weighted bin game. 
Recall that $M(s)=\operatorname{SzT}\left(T^{\alpha} s, \hat{b}(T) s+1\right)$. By the Szemerédi-Trotter Theorem we have

$$
M(s)=\operatorname{SzT}\left(T^{\alpha} s, \hat{b}(T) s+1\right) \leqslant C^{\prime}\left(\left(T^{\alpha} s\right)^{2 / 3}(\hat{b}(T) s+1)^{2 / 3}+T^{\alpha} s+\hat{b}(T) s+1\right)
$$

(where $C^{\prime}$ is the constant in the Szemerédi-Trotter Theorem).

Let $M^{\prime}(s)$ be the right hand side of this inequality. Since $M^{\prime} \geqslant M$, Maker can also play the weighted bin game $\left(\hat{b}, M^{\prime}, T\right)$ finishing with weight $W$ at least $\varepsilon n / 2$ in the last remaining bin. Now

$$
\begin{aligned}
\Delta M^{\prime}(s)= & C^{\prime}\left(T^{\alpha}\right)^{2 / 3}\left(((s+1)(\hat{b}(T)(s+1)+1))^{2 / 3}-(s(\hat{b}(T) s+1))^{2 / 3}\right) \\
& +C^{\prime} T^{\alpha}+C^{\prime} \hat{b}(T) \\
= & O\left(\left(T^{\alpha} \hat{b}(T)\right)^{2 / 3} s^{1 / 3}+T^{\alpha}+\hat{b}(T)\right) .
\end{aligned}
$$

Thus, Lemma 8 implies that the weight $W$ in the final bin of the weighted bin game $\left(\hat{b}, M^{\prime}, T\right)$ satisfies

$$
\begin{aligned}
W & =O\left(\frac{1}{\hat{b}(T)} \sum_{t=1}^{T} \frac{\left(T^{\alpha} \hat{b}(T)\right)^{2 / 3} t^{1 / 3}+T^{\alpha}+\hat{b}(T)}{t}\right) \\
& =O\left(\frac{1}{\hat{b}(T)}\left(T^{(2 \alpha+1) / 3} \hat{b}(T)^{2 / 3}+T^{\alpha} \log T+\hat{b}(T) \log T\right)\right) \\
& =O\left(\frac{1}{b(T)}\left(T^{(2 \alpha+1) / 3} b(T)^{2 / 3}+T^{\alpha} \log T+b(T) \log T\right)\right) .
\end{aligned}
$$

where the final line follows since $\hat{b}=\frac{\varepsilon}{4} b$ and $\varepsilon$ is fixed.

Finally, if $\alpha \geqslant 1$ and $b(t)=\omega(\log t)$, then since $T^{(2 \alpha+1) / 3} \leqslant T^{\alpha} \leqslant n$, Equation (**) implies that $W=o(n)$. Similarly, if $\alpha<1$ and $b(T)=\omega\left(T^{1-\alpha}\right)$ then from $(* *)$ we see that $W=o(n)$. In either case this contradicts $W \geqslant \varepsilon n / 2$.

\section{$5 \quad$ Proof of Theorem 3}

The rough idea is that Maker can follow the (implicit) strategy given for the weighted bin game by choosing several parallel lines, one corresponding to each bin. If at any point Breaker claims a point on one of these lines then Maker views that line as 'dead' and will choose not to play on that line again. The ideas of Lemma 8 suggest that in $T$ rounds Maker should be able to make a bin of weight roughly $m(T) \log T / b(T)=\Omega(m(T))$. Thus, for $T$ such that $m(T)=(1-\varepsilon) n$, Maker should have a strategy to get $\Omega(n)$ points on a line segment, after a turn of Breaker's, which (provided that this $\Omega(n)$ is at least $\varepsilon n$ ) means Maker wins by time $T+1$.

There are two problems with this argument: the first is that Lemma 8 is only an upper bound and the second is that in the weighted bin game Maker can place arbitrary weights 
in bins, but in the n-in-a-row game he has to claim an integer number of points on each line.

A short calculation (which we do below) solves the first problem, and a little care with the rounding solves the second.

Lemma 9. Let $m(t)=t^{\alpha}$ with $\alpha>0$ and $b=O(\log t)$. Then, for any given $T$, at the end of round $T$ (i.e., after Breaker's turn) Maker can guarantee to obtain

$$
\Omega\left(\frac{m(T) \log T}{b(T)}\right)
$$

points on an active line segment.

Proof. Let $r$ be the largest power of 2 less than $T / 2$. We consider only the rounds between time $T-r$ and $T$. During this period Maker is claiming at least $m(T / 2)$ points per turn, and Breaker is claiming at most $b(T)=O(\log T)$ points per turn.

With a slight abuse of notation let $m=\lfloor m(T / 2)\rfloor$ and $b=\lceil C \log T\rceil$. We show that, for $T$ sufficiently large, Maker can obtain an active line segment with at least $m(T) \log _{2}(T / 4) / 2^{\alpha+2} b(T)$ points at the end of these $r$ rounds while only claiming $m$ points each turn and allowing Breaker to claim $b$ each turn.

Maker's strategy is as follows. He picks $r b+1$ parallel horizontal lines of $n$ consecutive points, not containing any point that has already been claimed. Initially we will call all these lines live. If at any time during the next $r$ rounds Breaker claims a point in one of these lines, that line will become dead. However we will also allow Maker to designate some lines as dead himself. Note that a live line necessarily contains no point of Breaker's. Maker's strategy is to claim points so that the following two conditions hold at the end of each of his turns:

- Maker's points form an initial segment of each live line;

- The difference between the number of points Maker has claimed in any two live lines is at most 1 .

It is clear that there is a strategy that achieves this, explicitly, by always claiming the next point on any live line containing a minimal number of Maker's points.

During the first $r / 2$ rounds Maker claims $m r / 2$ points, and so each line that is still live at the end of these rounds contains at least

$$
\left\lfloor\frac{m r}{2(r b+1)}\right\rfloor \geqslant \frac{m}{4 b}
$$

points, where the inequality holds for all $T$, and thus $r$, sufficiently large.

During these $r / 2$ rounds, Breaker has claimed a point in at most $r b / 2$ lines, hence there are still at least $r b / 2+1$ live lines. Maker arbitrarily selects $r b / 2+1$ of these lines, and calls the rest dead. 
In the next $r / 4$ rounds Maker claims $m r / 4$ points, and so each line that is still live at the end of these rounds contains at least

$$
\frac{m}{4 b}+\left\lfloor\frac{m r}{4\left(\frac{r b}{2}+1\right)}\right\rfloor \geqslant 2 \times \frac{m}{4 b} .
$$

During this time Breaker has claimed a point in at most $r b / 4$ lines, and so there are still at least $r b / 4+1$ live lines. Again, Maker selects $r b / 4+1$ of these lines and calls the rest dead.

Maker repeats this $\log _{2} r$ times. At the end of these $r$ rounds the single live line will have at least $\frac{m}{4 b} \log _{2} r$ points on it.

Since $m=m(T / 2)=m(T) / 2^{\alpha}$ and $b=b(T)$ we have

$$
\frac{m}{4 b} \log _{2} r \geqslant \frac{m(T)}{2^{\alpha+2} b(T)} \log _{2}(T / 4)=\Omega\left(\frac{m(T) \log T}{b(T)}\right)
$$

as claimed.

The proof of Theorem 3 immediately follows from Lemma 9 and some simple calculation.

Proof of Theorem 3. Suppose that $\alpha, m$ and $b$ are as in the statement of the theorem. Fix $\varepsilon>0$ to be chosen later and let $T$ be such that $m(T+1)=(1-\varepsilon) n$. Since $b(T)=O(\log T)$, by Lemma 9 at the end of round $T$ Maker can obtain an active line segment with at least

$$
\Omega\left(\frac{m(T) \log T}{b(T)}\right)=\Omega(m(T))=\Omega(n)
$$

points. Thus, provided that we chose $\varepsilon$ less than the implicit constant in this ' $\Omega$ ', Maker can claim $(1-\varepsilon) n$ points on this active line segment on his next turn and win. Thus $\tau_{n} \leqslant T+1$ and hence $m\left(\tau_{n}\right) \leqslant(1-\varepsilon) n$, for all sufficiently large $n$.

For the final part suppose that $b(t)=o(\log t)$. Let $T=T(n)$ be a function to be defined later. By Lemma 9, Maker can obtain an active line segment containing

$$
\Omega\left(\frac{m(T) \log T}{b(T)}\right)
$$

points. Thus, since $b(t) / \log T=o(1)$, we can define $T$ such that $m(T)=o(n)$ but

$$
\frac{m(T) \log T}{b(T)}=\omega(n) .
$$

Therefore, in this case, Maker can obtain an active line segment with at least $n$ points by time $T$ such that $m(T)=o(n)$, even at the end of the Breaker's turn. 


\section{The directed and batched games for $\alpha<1$}

In this subsection we prove Theorem 4 giving the lower bound for the $(m, b)$-directedBreaker game.

Proof of Theorem 4. In fact we will show a stronger result: with $m$ and $b$ as in the statement of the theorem the batched version of the $(m, b)$-directed-Breaker game is a Maker win.

Indeed, Maker chooses to play for $T$ rounds, for some $T=T(n)$ we will define later. During this time in the normal game Maker would claim $\Omega\left(T^{1+\alpha}\right)$ points. In the batched game his strategy is to claim this many points as the integer points in a rectangle with sides $n$ and $\Omega\left(T^{1+\alpha} / n\right)$. This set contains $\Omega\left(T^{2+2 \alpha} / n^{3}\right)$ lines containing $n$ of Maker's points $\left(\Omega\left(T^{1+\alpha} / n\right)\right.$ starting points and $\Omega\left(T^{1+\alpha} / n^{2}\right)$ gradients $)$.

Breaker claims at most $b(T) T^{1-\alpha}=o\left(T^{2-\alpha}\right)$ points. Thus, we can define $T$ such that $m(T)=o(n)$, but the number of points Breaker has to claim is fewer than the number of lines Maker has. Hence, at least one of Maker's lines will not receive a point, and so, at the end of the game Maker will have an active line segment containing at least $n$ points. Therefore Maker wins the batched version of the directed-Breaker game as claimed.

Theorem 4 shows that the bound given by Theorem 2 in the range $\alpha<1$ is essentially tight for the $(m, b)$-directed-Breaker game. In fact we show that, when $b(t)=o\left(t^{1-\alpha}\right)$, not only can Maker win the game quickly, he can even do so in the batched version of the game, which is harder for him.

Our final result shows that this is not true for the $(m, b)$ - $n$-in-a-row game. Indeed, in the batched $(m, b)$-n-in-a-row game we have $m\left(\sigma_{n}\right)=(1+o(1)) n$ even when $b(t)$ is substantially smaller than the bound given in Theorem 2. Hence, if we hope to show that there is a matching lower bound in the case $\alpha<1$, such a strategy must make use of the turn based nature of the game.

Proposition 10. Suppose that $\alpha<1, m(t)=t^{\alpha}$ and $b(t)=\omega(\log t)$. Then the winning time $\sigma_{n}$ of the batched $(m, b)$-n-in-a-row game is such that $m\left(\sigma_{n}\right)=(1+o(1)) n$.

Proof. Let $\varepsilon>0$ and suppose that Maker chooses to play for $T$ rounds for some $T$ with $m(T) \leqslant(1-\varepsilon) n$. We will show that Breaker wins for all sufficiently large $n$.

Suppose that Maker has already claimed his points. Observe that we may assume that $T(n)=\omega(1)$ since otherwise Maker does not claim $n$ points in total and cannot possibly win. Also, note that the constraint on $m(T)$ implies that $T^{\alpha} \leqslant n$.

We will show via a probabilistic argument that Breaker can choose his points such that Maker has no active line segment with more than $\varepsilon T^{\alpha} \leqslant \varepsilon n$ points. Let $A$ be a subset of Maker's points chosen at random, by including each of Maker's points in $A$ independently with probability $p=\frac{2 \log T}{\varepsilon T^{\alpha}}$.

Maker claimed at most $T^{1+\alpha}$ points, and so, by the Szemerédi-Trotter Theorem, Maker has at most

$$
O\left(\frac{\left(T^{1+\alpha}\right)^{2}}{\left(\varepsilon T^{\alpha}\right)^{3}}+\frac{T^{1+\alpha}}{\varepsilon T^{\alpha}}\right)=O\left(T^{2-\alpha}\right)
$$


(since $\alpha<1$ ) line segments with more than $\varepsilon T^{\alpha}$ points in them. (As in Section 2 we are considering any line segments containing $\approx k \varepsilon T^{\alpha}$ points as $\lceil k\rceil$ line segments each containing at most $\varepsilon T^{\alpha}$ points.)

The probability that the set $A$ contains no point from a line segment of length $\varepsilon T^{\alpha}$ is $\left(1-\frac{2 \log T}{\varepsilon T^{\alpha}}\right)^{\varepsilon T^{\alpha}} \approx T^{-2}$. Hence the probability that there exists such a line segment that does not receive a point from $A$ is $O\left(T^{2-\alpha} \times T^{-2}\right)=O\left(T^{-\alpha}\right)$ which is less than $1 / 10$ for all $n$, and thus $T$, sufficiently large.

The probability that the set $A$ contains at most $p T^{1+\alpha}=\frac{2}{\varepsilon} T \log T$ points is approximately $1 / 2$. Thus, with positive probability, the set $A$ contains at most $\frac{2}{\varepsilon} T \log T$ points and contains a point from every one of Maker's line segments of length at least $\varepsilon T^{\alpha}$. In particular, there exists a set $A^{\prime}$ satisfying both these conditions.

Now, Breaker gets to claim $B=\sum_{t=1}^{T} b(t)$ points. Since $b(t)>\frac{4}{\varepsilon} \log t$ for all sufficiently large $t$ we see that $B>\frac{2}{\varepsilon} T \log T$ for all sufficiently large $T$ (and thus for all sufficiently large $n$ ).

Thus, Breaker's strategy is to pick the set $A^{\prime}$ given above. This strategy wins, which shows that $m\left(\sigma_{n}\right) \geqslant(1-\varepsilon) n$.

Remark. We note that, by a similar argument to Proposition 5 , for all $\alpha \geqslant 1$, if $m(t)=t^{\alpha}$ and $b(t)=\omega(1)$ then the winning time $\sigma_{n}$ for the batched $(m, b)$-n-in-a-row game is such that $m\left(\sigma_{n}\right)=(1+o(1)) n$.

\section{Extensions of the results}

Although we have stated and proved the results in $\mathbb{Z}^{2}$ they hold in rather more generality. Indeed, since the proof of Theorem 2 only relies on the Szemerédi-Trotter Theorem, it also holds, for the analogous game, wherever the Szemerédi-Trotter Theorem holds. In particular, it holds in higher dimensions (with the winning sets being lines), in $\mathbb{Z}^{d}, \mathbb{Q}^{d}$ and $\mathbb{R}^{d}$, and in cases where the winning sets are more general curves: for example solutions to polynomial equations, or any other curves any two of which only intersect in a bounded number of places. In particular if $\alpha \geqslant 1$ then Breaker can win any of these games whenever $b(t)=\omega(\log t)$.

\section{Open questions}

In the case where $\alpha \geqslant 1$ there is a clear threshold between a Maker win and a Breaker win, however when $\alpha<1$ the bounds are still very far apart. Our key question is to find the correct bound in this case. Define the threshold function

$$
\beta_{c}(\alpha)=\sup \left\{\beta: \begin{array}{l}
\text { The }(m, b) \text {-n-in-a-row game with } m(t)=t^{\alpha} \\
\text { and } b(t)=t^{\beta} \text { is a Maker win. }
\end{array}\right\} .
$$

Question 1. Find $\beta_{c}(\alpha)$ for $\alpha<1$.

Our lower bound for $b(t)$ in this case is only logarithmic so we do not even know the answer to the following simpler question. 
Question 2. Is $\beta_{c}(\alpha)>0$ for any $\alpha$ ?

The form of our bounds seem to suggest that as $\alpha$ increases Breaker can win with fewer and fewer points. Indeed our upper bound for $b$ is monotone decreasing. Perhaps the actual threshold is also monotonic?

Question 3. Is $\beta_{c}(\alpha)$ monotone decreasing?

We saw in the previous section that the results generalise to many other settings. In many senses the most natural setting for our result is $\mathbb{Q}^{2}$ rather than $\mathbb{Z}^{2}$. We have seen that for Maker to win before time $T=(1-\varepsilon) n$, he must guarantee to have a line segment with at least $\varepsilon n$ points on it after Breaker has played. In $\mathbb{Q}^{2}$ in the case $m(t)=b(t)$ we do not even know that Maker can ever guarantee to have such a line segment.

Question 4. Fix $n$ and suppose that the $(m, b)$-n-in-a-row game is played on $\mathbb{Q}^{2}$ with $m(t)=b(t)=t$. Can Maker guarantee to have a line segment containing $n$ points after Breaker's move? If so, by what time can he guarantee to have such a line segment?

Another extension where we don't know the answer is the following: suppose we say instead that Maker wins if at any point he has claimed a set of $n$ points whose convex hull contains none of Breaker's points. Note that the winning sets in the $(m, b)$-n-in-a-row game are of this form, and so this game is easier for Maker than the $(m, b)$-n-in-a-row game.

Question 5. Let Maker's family of winning sets be sets of $n$ points in $\mathbb{Z}^{d}$ (alternatively $\left.\mathbb{Q}^{d}\right)$ whose convex hull contains none of Breaker's points. What is the threshold for $b(t)$ when $\alpha=1$ ? In particular can Maker win this game with $m(t)=t$ and $b(t)=t^{\varepsilon}$ for some $\varepsilon>0$ ?

\section{References}

[1] J. Beck. On a generalization of Kaplansky's game. Discrete Mathematics, 42(1):2735, 1982.

[2] J. Beck. Combinatorial Games: Tic-Tac-Toe theory. Cambridge University Press, 2008.

[3] J. Erde. An n-in-a-row game. Integers, 15, 2015.

[4] D.J. Kleitman and B.L. Rothschild. A generalization of Kaplansky's game. Discrete Mathematics, 2(2):173-178, 1972.

[5] E. Szemerédi and J. R. Trotter. Extremal problems in discrete geometry. Combinatorica, 3(3-4):381-392, 1983.

[6] T. G. L. Zetters. 8 (or more) in a row. The American Mathematical Monthly, 87(7):575-576, 1980. 